\title{
Entretien
}

\section{Bertrand Vissac ou les chemins de traverse d'un chercheur citoyen}

\section{Propos recueillis par François Papy}

\author{
Bertrand Vissac ${ }^{\mathrm{a}}$, François Papy ${ }^{\mathrm{b}}$ \\ a Zootechnicien, INRA, France \\ b Agronome, INRA, INA P-G, UMR SADAPT, Bât. Eger, BP 1, 78850 Thiverval-Grignon, France
}

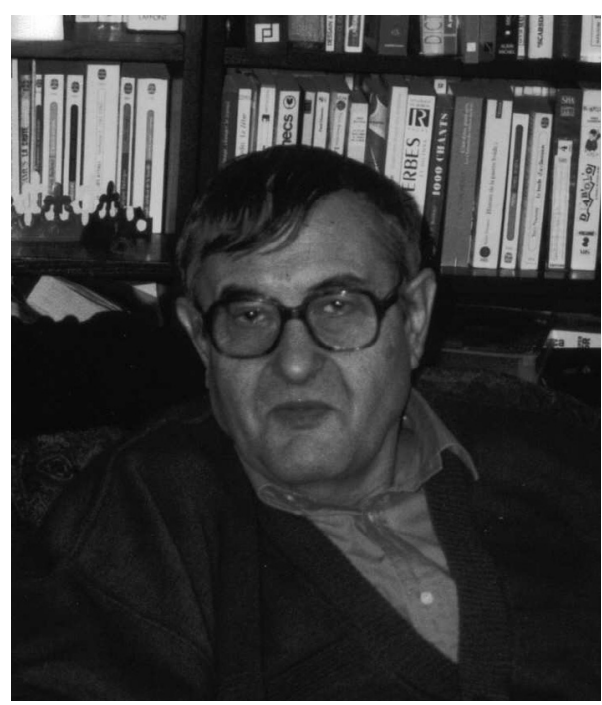

Au moment où nous relisons les épreuves, nous apprenons avec beaucoup de tristesse le décès de Bertrand Vissac, le mercredi $1^{\mathrm{er}}$ septembre. Cela donne encore plus de poids à ce texte passionnant.

La Rédaction

\section{Biographie}

Bertrand Vissac, né le 14 janvier 1931 à Langeac (Haute-Loire), était ingénieur agronome de l'INA P-G, et directeur de recherche émérite de l'Inra. C'est dans cet établissement de recherche qu'il a fait sa carrière, d'abord au laboratoire de zootechnie de l'INA, puis au Centre national de zootechnie à Jouy-en-Josas. Il a participé à la mise en place des schémas de sélection collective des éleveurs de bovins et du Centre national de traitement de l'information de génétique animale de Jouy. Il a ensuite occupé les fonctions de chef du département de Génétique animale (1972-1980) puis du département Systèmes agraires et développement (1980-1994). C'est ainsi que, collaborant avec d'autres sciences biotechniques et des sciences humaines, il a emprunté beaucoup de "chemins de traverse ». Il en expose les raisons dans l'ouvrage édité en 2002, avec le concours de Bernadette Leclerc: Les Vaches de la République: saisons et raisons d'un chercheur citoyen (INRA Éditions).
François Papy (NSS) : À l'heure où il faut «sauver la recherche ", quel meilleur moyen pour les chercheurs de montrer que, comme tout un chacun, ils sont aussi des citoyens; et quel meilleur exemple de recherche citoyenne que la vôtre. Le sous-titre de l'ouvrage que vous avez publié en 2002 le dit bien : Les Vaches de la République: saisons et raisons d'un chercheur citoyen. Vous y décrivez vos emballements, vos doutes, vos questions au cours d'une carrière qui a accompagné l'énorme bouleversement de l'agriculture française depuis la dernière guerre. Autour du concept de génération animale, vous reliez l'évolution des sociétés humaines à celle des populations animales. Puisque les réflexions que vous développez

Auteur correspondant : F. Papy, papy@grignon.inra.fr dans cet ouvrage suivent les chemins de traverse que vous avez pris, je souhaiterais, pour commencer, vous interroger sur votre début de carrière, lorsque vous êtes entré à l'Inra comme chercheur en sélection animale.

Bertrand Vissac : Ma carrière de zootechnicien a connu quelques prémisses par l'immersion dans l'élevage des vaches, dès mon plus jeune âge : c'était un élevage paysan quasi autarcique dans les années de la guerre 1939-1945. Ce n'est qu'incité et aidé par ma famille que j'ai pu entrer à l'Agro; mais mon classement de sortie ne m'a pas permis d'accéder aux postes offerts par l'Inra, alors en cours création, et qui attirait les meilleurs. J'avais convolé en justes noces dès la deuxième année d'études, ce qui n'avait pas favorisé mon classement. Mon seul titre de gloire final était la réussite au concours de chef 
de centre d'insémination que devaient passer les élèves de la section «Agriculture et élevage ». Fatigué par mes études, j'ai été ensuite ajourné, puis réformé de l'armée deux ans plus tard. C'est dans cette situation d'incertitude familiale que j'ai rencontré Charlet, puis Poly ${ }^{1}$ qui va beaucoup marquer la suite de ma carrière. Recruté par l'Inra pour sélectionner les animaux, ce dernier avait été affecté au labo du professeur Leroy, fondateur de la zootechnie française et de la Fédération européenne de zootechnie (FEZ). Il s'agissait, pour les responsables de l'Inra, de ménager la susceptibilité du professeur Leroy après le départ de ses premiers élèves zootechniciens pour une affectation dans le nouveau centre de Jouy-en-Josas. Ce Centre national de la recherche zootechnique était alors le phare de la science animale qui drainait tous les crédits et présentait une image d'avenir. J'ai donc été recruté pour aider Poly comme agent technique principal avant d'intégrer le cadre scientifique et de suivre la carrière de ce corps à l'Inra.

F.P. : Où la sélection animale en était-elle à cette époque?

Bertrand Vissac : La sélection animale était alors surtout basée sur l'observation de la conformation des animaux; le contrôle laitier était peu pratiqué et servait surtout, depuis l'Agro, à valider l'alimentation des animaux et les activités des vachers des exploitations de la région parisienne. J'ai donc commencé à m'intéresser d'abord à ces épreuves subjectives pour tenter de les améliorer par des mensurations corporelles et pour évaluer leur valeur prédictive des performances laitières et bouchères des animaux. Ces travaux, faits dans des fermes et des abattoirs, n'ont jamais été publiés ; ils ont surtout servi à montrer notre souci de ne pas rejeter d'emblée les pratiques traditionnelles de la sélection. Mais peu à peu, grâce au démarrage de l'insémination artificielle des bovins, à la vulgarisation de la génétique quantitative, puis à l'apparition des premiers ordinateurs d'usage convivial, il nous a semblé que le moment était venu de lancer les premières opérations expérimentales de contrôle de descendance des taureaux d'insémination artificielle. Jusqu'alors ces taureaux étaient choisis dans la descendance des premiers prix des « concours de beauté » qu'étaient les concours de race. Vaste débat! Car nos travaux heurtaient de front les intérêts des sélectionneurs de l'ancienne école, amis du professeur Leroy, qui se voyaient ainsi privés à la fois d'une compétence de jugement et du marché lucratif des taureaux de monte naturelle qui lui était attaché. Ce conflit nous a conduits à quitter l'Agro précipitamment. Mais de mon passage rue

\footnotetext{
1 Pierre Charlet était à l'époque maître de conférences à la chaire de Zootechnie de l'Agro et Jacques Poly, chargé de recherche Inra au laboratoire de recherche annexé à la chaire de Zootechnie. Il allait devenir plus tard chef du département de Génétique animale de l’Inra, puis président directeur général de cet institut.
}

Claude Bernard, j'ai été durablement imprégné par l'esprit d'une recherche associant les chercheurs avec les éleveurs dans leurs exploitations. Cette attitude, à l'opposé de l'esprit du centre de recherche de Jouy-en-Josas, intéressait la direction de l'Inra qui craignait, en effet, le coût excessif des moyens expérimentaux d'un futur département de Génétique animale et ses conséquences pour les autres disciplines déjà en place et particulièrement gourmandes dans leur phase d'installation. C'est dans ce contexte que, dans les fermes, des opérations expérimentales de testage des taureaux ont été mises en place, peu à peu, dans différentes régions par les chercheurs généticiens, rassemblés à Jouy sous la houlette de Poly.

F.P. : Vous avez opposé le travail que vous faisiez avec Poly à la conception de l'amélioration qu'avait le professeur Leroy. Si j'ai bien compris, vous avez travaillé avec le contrôle laitier dans des régions où il n'y avait pas de livres généalogiques. N'était-ce pas déjà une option de travailler avec certains éleveurs, alors que le professeur Leroy travaillait avec d'autres : les grandes familles d'éleveurs qui, gérant ces livres, organisaient les races?

Bertrand Vissac : En fait nous n'avons pas négligé d'étudier les pratiques traditionnelles de ces derniers, mais le conflit entre leurs pratiques subjectives et le testage collectif paraissait incontournable. Tout naturellement les collaborations se sont ouvertes avec des centres d'insémination dont les zones d'action ne comportaient pas de ténors des races et qui étaient assez éloignés de leurs «berceaux » d'origine. Heureusement que le ministère a accepté de choisir des taureaux de centres d'insémination en fonction des tests que nous avions mis en place et non pas seulement sur les critères de beauté de la race. Nous avons ainsi travaillé dans l'Yonne, le Loiret ou le Jura pour les races laitières, dans l'Aveyron et le Tarn pour les races à viande. Les bases sociale et géographique de nos recherches élargissaient ainsi considérablement le champ d'action du professeur, concentré dans les berceaux de race du Bassin parisien, haut lieu du recrutement des premiers élèves de l'Agro. Avec l'appui du tandem jurassien, Edgar Faure et Jacques Poly devenu son conseiller, le général de Gaulle a eu la clairvoyance de profiter de cette dynamique pour lancer une grande action en direction des campagnes françaises. Le choix d'une loi pour promouvoir et organiser une sélection collective par les mâles se justifiait en effet par le niveau embryonnaire et l'état de délabrement des organismes français de sélection, qu'il s'agisse des livres généalogiques ou des syndicats de contrôle laitier. Parce qu'ils étaient fondés sur la libre entreprise, en Angleterre, ou sur des organisations coopératives locales partiellement autofinancées, aux Pays-Bas, les principes de la sélection chez nos voisins européens n'étaient, en effet, pas adaptés à nos conditions. Une loi paraissait donc nécessaire ; elle devait respecter le cadre républicain du département et permettre à la fois la constitution de puissantes 
organisations techniques concurrentielles sans marginaliser des éleveurs ou des zones isolées. C'est donc à une quasi-unanimité que cette loi a été votée par des députés conscients de la nécessité de rendre l'élevage français compétitif et qui, selon leur tendance politique, regardaient les ranchs américains ou les kolkhozes russes que donnait en référence la presse de l'époque. Les bovins ont donc été le support d'opérations collectives que les autres espèces imiteront plus tard.

F.P. : Vos travaux de l'époque ont donc marqué fortement cette loi sur l'élevage de 1966.

Bertrand Vissac : C'était d'abord l'œuvre de Poly, même s'il utilisait habilement le combustible que constituaient nos travaux sur les schémas de sélection pour soumettre sa loi au «feu de la critique ». L'originalité que nous avons eue avec Marcel Poutous ${ }^{2}$ a surtout consisté à rompre avec l'esprit des recherches de génétique appliquée qui visaient à optimiser les conditions de sélection pour maximiser le progrès génétique sur les quantités de lait ou de viande. Tel était le sens du message de la productivité. Mais la situation catastrophique de l'élevage français et les réticences du ministère à alimenter une vulgarisation, considérée comme un véritable «tonneau des Danaïdes » des crédits publics, nous ont poussés à intégrer dans ces recherches d'optimisation les coûts et les recettes de la sélection. Nous étions dans la droite ligne des opérations de rationalisation des choix budgétaires de l'époque. Ces travaux prenaient aussi en compte les frais financiers liés aux énormes temps de latence de la sélection sur descendance; ils aboutissaient à des revenus du capital parfaitement convaincants tant pour les jeunes agriculteurs que pour les inspecteurs des finances : on était en présence d'une grande machinerie qui, à l'image d'EDF, couvrait parfaitement les besoins nationaux dans le sens de la politique et qui engageait l'avenir sur des décennies. Certes, on ne prenait pas en compte les hypothèses de saturation des marchés : qui se posait, dans les années 1960, la question des limites des débouchés laitiers ou des déséquilibres entre le lait et la viande? Les immenses besoins du tiers-monde étaient censés absorber les excédents, sans que personne ne se pose la question de leur solvabilité.

F.P. : Dans votre livre, vous dites avoir très vite émis des réserves vis-à-vis de ce progrès génétique. Comment avez-vous pris conscience des limites de ce progrès? S'agit-il d'un virage dans votre carrière? Si oui, comment s'est-il amorcé après la phase de recherche qui a abouti concrètement à la loi sur l'élevage?

Bertrand Vissac : Les projets collectifs contenus dans la loi reposaient sur un postulat de spécialisation entre des races laitières, traites, et des races à viande, allaitant leur veau. Or notre production de viande était surtout

\footnotetext{
2 Alors chargé de recherche Inra au laboratoire de recherche annexé à la chaire de Zootechnie de l'Agro.
}

assurée par le cheptel laitier à travers des races mixtes, lait-viande. Le postulat moderniste faisait ainsi fi des équilibres génétiques entre les aptitudes laitière et bouchère des races traites et conduisait à une élimination progressive des races mixtes au profit de la Holstein. Un déséquilibre commercial va ainsi apparaître dans la décennie 70 entre une production de lait excédentaire et une production de viande déficitaire. Dès le départ, avant même la loi sur l'élevage, nous avions prévu de pallier en partie les inconvénients de cette spécialisation industrielle en imaginant de créer des souches mâles de race à viande pour améliorer par croisement de première génération la valeur bouchère des veaux de vaches de race mixte. Ceci permettait d'exploiter des vaches qui allaient disparaître, tout en assurant à de vieux éleveurs une fin de carrière correcte!

Ces oppositions génétiques entre caractères prenaient un relief particulier dans le cas d'une race rustique comme l'Aubrac. Race à triple fin : lait-viande-travail, elle se trouve limitée vers 1960, du fait de l'arrêt de la traite et de l'attelage des bœufs, à une stricte fonction bouchère vers laquelle sa conformation ne la désigne guère. Toujours est-il que les prouesses attendues de la spécialisation amènent, en 1965, les fonctionnaires bruxellois à conclure à l'inutilité de ce type de race, inadaptée à leurs schémas; ils décident de "geler » leur territoire d'élevage. Avec Béranger ${ }^{3}$ et quelques autres, je me trouve alors engagé dans une opération de recherche du CNRS apparemment surréaliste : la recherche coopérative sur programme (RCP) de l'Aubrac. D'un côté, des chercheurs ethnologues et muséologues veulent inscrire dans les archives d'un musée la société aubracienne avant qu'elle ne disparaisse, mais, d'un autre côté, la dynamique des éleveurs a tôt fait de nous convaincre de l'intérêt d'un projet d'élevage bovin extensif sur de telles étendues herbeuses, si rares en Europe. L'idée était d'autant plus séduisante que les alternatives envisagées au gel des terres étaient irréalistes : sur ce plateau venté, la forêt ne poussait pas et son enneigement était insuffisant pour assurer une saison de ski. C'est ainsi qu'a été conçu et adopté par les éleveurs le croisement de la petite vache Aubrac avec des taureaux charolais, un croisement qui donnait, au sevrage, des veaux presque aussi gros mais mieux conformés que leur mère. Le mariage des muséologues et des zootechniciens ne faisait ainsi que traduire le besoin d'allier la tradition et la modernité dans la mise en œuvre du changement technique : les écomusées de G.-H. Rivière ${ }^{4}$ sont nés là de ce besoin. C'est là que je me suis posé la question des limites de notre orientation vers la productivité.

${ }^{3}$ Claude Béranger était alors chargé de recherche à l'Inra ; il devait bien plus tard être directeur scientifique du développement, en charge du département Systèmes agraires et développement.

${ }^{4}$ Georges-Henri Rivière est le fondateur du musée des Arts et Traditions populaires. 
C'était l'époque où le Club de Rome et la conférence de Stockholm faisaient prendre conscience à l'opinion de la limitation des ressources minérales et de l'importance de la conservation des ressources biologiques. La RCP Aubrac montrait alors que cette question concernait autant les espèces domestiques que les espèces sauvages. Elle fournissait même une sorte de modèle qui dépassait la conservation dans un milieu figé pour l'orienter dans une direction prospective intégrant un patrimoine passé avec des projets d'avenir assumés par l'établissement actuel des éleveurs. Cette idée rebondira 10 ans plus tard quand il faudra répondre aux crises du pétrole et du soja.

F.P. : Le concept de génération animale que vous utilisez dans votre livre implique l'existence de modèles de conduite des populations animales. Comment les chercheurs ont-ils élaboré ces modèles?

Bertrand Vissac : Ainsi que je l'ai dit, dès mon entrée à l'Inra, j'ai été engagé dans une dynamique de transformation des méthodes d'amélioration génétique. L'expression de génération animale est parfaitement adaptée pour désigner nos préoccupations de l'époque. Aristote l'utilisait déjà dans l'Antiquité pour caractériser à la fois la reproduction (l'acte de chair), l'hérédité des caractères et l'influence du milieu sur les performances : un ensemble de phénomènes qu'on ne savait pas dissocier alors. Or, au lendemain de la guerre de 39-45, si on connaissait les grandes lignes des bases biologiques de ces composantes qui allaient structurer les disciplines de l'Inra, on ne savait, alors, ni les maîtriser ni les recombiner. Dans ce contexte, j'ai donc été confronté à un renouvellement considérable des modèles qui sont à la base de la construction des connaissances sur le vivant.

Le premier type de modèle était un modèle incarné par un homme de génie : il est généralement admis que c'est Bakewell qui, parmi l'élite des agronomes anglais du $\mathrm{XVIII}^{\mathrm{e}}$ siècle, a fait figure de fondateur de la zootechnie moderne. Il a défini les grandes lignes des schémas de sélection et leur mise en œuvre par des réseaux d'éleveurs dont il était la tête pensante et agissante pour répondre aux besoins du marché londonien. Ce modèle subjectif a ensuite été codifié par le système élitiste des livres généalogiques à travers des tables de pointage que nous avons tenté de valider.

Lorsque la recherche agronomique a été créée en France, l'état des connaissances scientifiques a paru suffisant pour s'appuyer sur des modèles analytiques quantifiés, limités au départ à un ou deux caractères et au choix des mâles, mais avec l'espoir raisonnable d'affiner cette procédure descendante ou top down d'amélioration de la productivité individuelle.

Nous nous sommes retrouvés dans les années 19651970 avec un point fort : nous avions mis en place des schémas de sélection efficaces avec des éleveurs, mais sans jamais avoir expérimenté sur aucun animal dans un domaine expérimental. La question se posait de valider ce qu'on faisait dans des fermes sur d'autres caractères que la production laitière, vérifier jusqu'où on pouvait aller; n'avions-nous pas, en augmentant la production laitière, entraîné des détériorations d'autres qualités? Dans la sélection d'animaux à forte musculature pour le croisement de première génération, ne pouvait-on pas utiliser des animaux culards? Ce sont les éleveurs et les marchands de bestiaux de Saône et Loire qui nous ont poussés à étudier le caractère culard, car ils introduisaient illégalement dans les fermes les taureaux porteurs de ce qui était officiellement considéré comme une anomalie et ils concurrençaient ainsi l'insémination artificielle. La création de domaines expérimentaux au Pin au Haras, à Bourges, à Carmaux a ainsi permis de valider, d'affiner, de complexifier les modèles généraux d'amélioration génétique.

Mais nous ne pouvions cependant pas approcher suffisamment la complexité du réel par la seule expérimentation. Vers 1970, inspirés par nos collègues du Texas, nous nous sommes lancés dans «l'analyse de système » : l'objectif était de décomposer la complexité pour optimiser, par voie expérimentale, des croisements entre races de format et de musculature différents, soumises à des régimes alimentaires variés. Cela se pratiquait, alors, au Texas, dans des exploitations de 20000 ou 50000 vaches où on essayait de savoir s'il fallait utiliser des vaches de petit, moyen ou grand format, les élever en race pure ou les croiser et jusqu'où les croiser... Nous essayions d'imiter ces expériences faites au Texas chez nous, dans des domaines expérimentaux, à Bourges et ailleurs, mais il est très vite apparu que ce n'était pas transposable dans les conditions de l'élevage français de l'époque, qui était constitué de petits troupeaux, exploitant des surfaces relativement limitées et regroupés dans des villages - il $\mathrm{y}$ avait d'ailleurs de moins en moins de troupeaux par village. Cette « analyse de système » apparaissait donc comme totalement insuffisante. Ce réel complexe ne peut se décomposer a priori pour être reconstruit ensuite sur le papier.

F.P. : Pour continuer à travailler en scientifique sur ces questions, comment vous êtes-vous débrouillé, puisqu'il y avait une sorte d'obstacle expérimental dû à la faible taille des troupeaux et à la longue durée de la génération animale? Comment avez-vous continué à remettre en cause les premiers modèles que vous aviez élaborés et à en concevoir d'autres?

Bertrand Vissac : En Aubrac, comme dans d'autres opérations conduites ultérieurement en France ou à l'étranger, j'ai été confronté à des problèmes complexes échappant à nos objectifs et à nos modèles. En Corse, comme en Côte d'Ivoire ou à la Réunion, la productivité animale n'est pas au centre des préoccupations ; les finalités de l'élevage sont multiples et les acteurs sont associés dans des réseaux de relation, souvent opaques. Les problèmes émanant du terrain doivent donc être traduits en 
questions de recherche grâce à la collaboration de plusieurs disciplines de sciences de la nature et de sciences humaines : c'est plus difficile que de se contenter de questions qui ne tiennent pas compte du contexte de mise en œuvre. Dans cette veine, nous avons mis en œuvre des procédures de recherche-action où nos projets de recherche étaient associés dans le temps avec des projets de partenaires acteurs, chacune des deux parties adoptant le rythme nécessaire à l'exercice de son activité. Des modèles précédents, nous passions ainsi à une modélisation systémique dont la construction impliquait la recherche dans un «établissement humain » à base d'élevage. C'est en Corse que cette modélisation systémique a été pour la première fois conçue et mise à l'épreuve en intégrant la race animale et la société locale d'éleveurs transformateurs de lait ou de cochonnailles.

F.P. : Avant la Corse, il semble que c'est votre expérience en Aubrac qui est à l'origine de votre démarche de recherche-action, dont on parle tant maintenant. Comme, pour être reconnu, ce type de recherche doit être clairement explicité, j'aimerais bien que vous développiez comment vous l'avez progressivement mis en œuvre.

Bertrand Vissac: En Aubrac, en 1965, nous étions bien dans l'esprit de la recherche-action sans en avoir formalisé la méthode. On était une bande de copains, identifiés par leur discipline et, depuis cette époque, parfaitement complices. Chacun peut raconter la même histoire à sa façon sans incohérence. En ce qui me concerne, en tant que généticien, je voudrais d'abord fixer la mesure des temporalités de cette recherche-action sur la génération des bovins dans ces montagnes. Il faut savoir que l'intervalle de génération (âge moyen des animaux quand naissent leurs descendants) est de l'ordre de 8 ans pour les bovins ; il y a donc environ 4 générations de bovin par génération humaine. Le travail sur le terrain a en fait duré 3 à 4 ans avec une enquête de situation 10 ans après. Au début, en 1965, je me trouvais associé à des chercheurs d'autres disciplines (zootechnie, économie); ils étaient convoqués, comme moi, par un ethnologue (Corneille Jest) qui avait fait l'inventaire des 300 montagnes (lieux d'estive stables depuis la Révolution) et de leurs exploitations d'hivernage, ainsi que de la parentèle locale et parisienne des descendants de «bougnats » qui accueillaient les vachers dans les cafés pendant la morte-saison. Face à la disparition de la traite et de l'attelage des bœufs, nous avons accompagné les réflexions des éleveurs déjà fortement engagés dans un premier croisement viande; les comparaisons expérimentales des races paternelles au centre de sélection de Soual ont confirmé l'intérêt du croisement charolais. Ce croisement était déjà à l'œuvre sur le plateau sous la conduite des deux conseillers généraux, promus éleveurs et vendeurs de taureaux de monte naturelle pour aider leurs électeurs mais aussi conforter leur image auprès d'eux. Deux questions vont se poser dans les 10 années qui ont suivi :

- Comment finir le «maigre» en Aubrac? Comme on l'a vu, le croisement entre la petite vache rustique et le taureau charolais va d'abord être une véritable aubaine pour les naisseurs, mais aussi pour les acheteurs de maigre du Bassin parisien et d'Italie. Les Aubraciens n'avaient pas, en effet, assez de ressources fourragères pour assurer cette finition. La prolongation de l'élevage pour capter la plus-value de finition sur place restait néanmoins un premier objectif nécessitant de définir les âges et modalités d'élevage et de commercialisation des animaux, puis de les intégrer ensuite dans les systèmes d'élevage des exploitations selon leurs disponibilités et leur configuration foncière.

- Comment conserver une population de vaches Aubrac? La pratique du croisement était au départ tellement avantageuse qu'elle risquait de compromettre ensuite le renouvellement des femelles Aubrac. On a alors cherché à comparer les avantages du croisement suivant les pratiques d'élevage des vaches. Nous avons pu ainsi définir des zones plus favorables à l'élevage d'Aubrac en race pure, en même temps que nous mettions au point, pour profiter de l'effet de vigueur que procure l'hybridation, le croisement avec des mâles charolais. En fait, ce sont, plus tard, les crises de l'énergie puis du soja qui vont stimuler l'élevage des génisses Aubrac pures dans les zones de conquête du Sud-Ouest et du Midi.

Les réponses à ces deux questions en ont entraîné d'autres. On a assisté à un double mouvement d'intensification des exploitations et d'adaptation des zones de la montagne et du pourtour de l'Aubrac (Viadène, Boraldes, montagne aveyronnaise et lozérienne, vacheries transhumantes du causse Comtal) : élevage et finition des animaux pour la boucherie d'une part, renouvellement des femelles de la race d'autre part. Un formidable équipement des fermes en est résulté, avec une main d'œuvre de plus en plus rare et surchargée.

Parallèlement à cette orientation viande qui réduit le peuplement humain du massif et n'exploite pas l'originalité fromagère de la race, des éleveurs décident, enfin, de relancer la traite à partir d'une race fromagère, plus productive que l'Aubrac, originale pour la région : la Fleckvieh bavaroise, qui a absorbé la Pie rouge de l'Est. Plutôt que de lutter avec la fourme du Cantal, ils ont l'astuce d'exploiter le sous-produit de la fourme, l'aligot, comme produit commercial d'appel. On retrouve dans cette réaction des éleveurs de Viadène (zone favorable du Nord de l'Aubrac) les mêmes soucis d'intégration de la tradition avec la modernité et aussi d'authentification de leur matériel animal et de leur produit.

Autour de son pôle, Laguiole, l'Aubrac est ainsi devenu au plan national un modèle de conservation puis de promotion des races associées aux cultures locales dans la dynamique du développement rural. Cette histoire illustre bien l'accompagnement par la recherche des 
questions de développement posées par les éleveurs face à un contexte qui évolue.

F.P.: Votre expérience de recherche qui, dès le début de votre carrière, s'est faite en étroite collaboration avec les éleveurs, puis, plus tard, votre rencontre avec les sciences sociales, notamment en Aubrac, vous ont prédisposé à être le chef du nouveau département Systèmes agraires et développement $\left(\mathrm{SAD}^{5}\right)$ qui a été créé, en 1979, à l'Inra. Il serait intéressant de vous entendre sur l'interdisciplinarité : comment les disciplines sociales et techniques peuvent-elles collaborer efficacement? Quelles difficultés rencontrent-elles pour le faire?

Bertrand Vissac: Dans la RCP Aubrac une forme d'interdisciplinarité a été effectivement mise en œuvre : l'ethnologie a questionné les chercheurs agronomes, zootechniciens et technologues, puis a conduit les sociologues et géographes à étudier la recomposition de cet établissement humain qui a ainsi sauvé la région du gel des terres. Quand le département SAD a été créé, j'ai senti un sentiment de frustration : le sélectionneur de vaches ne retrouvait pas ses marques dans des recherches que ce département conduisait sur les systèmes agraires locaux considérés comme des assemblages fonctionnels de parcelles et de haies, d'exploitations, de bassins versants et de paysages. J'ai néanmoins respecté ce choix d'échelles qui était bien un choix fondateur indispensable. Mais je me suis questionné sur ce qui faisait, pour moi, la spécificité des races animales et de leurs systèmes agraires. J'ai d'abord invoquél'échelle géographique étendue attachée à l'élevage des populations de vaches. Mais je pense aujourd'hui que c'est le temps long, celui de l'histoire, qui associe, à travers l'élevage, des gènes et des pratiques collectives. Ce temps-là implique des recherches qui s'inscrivent dans l'évolution biologique et la culture des sociétés : qu'est-ce qu'une race animale si ce n'est le produit, à la fois volontaire et fruit du hasard, des rapports entre les gènes des animaux et la culture des hommes. Ce lien revêt un caractère particulier pour une espèce à long intervalle de génération comme les bovins, qui constituent de plus le liant biologique qui a construit nos systèmes agraires. Ce que j'avais sous les yeux en faisant mes recherches depuis Jouy et l'Aubrac entre 1960 et 1975 n'était de plus en plus pour moi que le déroulement de deux siècles de l'histoire de l'entrée de l'élevage bovin dans la modernité.

F.P. : Je comprends pourquoi votre ouvrage a une telle dimension historique!

Bertrand Vissac : Le point de départ de la modernité, ce sont donc les bourgeois terriens anglais qui l'ont donné en appliquant en agriculture les principes de l'industrie, et ce, dès le milieu du XVIII ${ }^{\mathrm{e}}$. Ils utilisent pour cela deux principes : intensifier d'abord la production en éliminant

\footnotetext{
${ }^{5}$ Ce département s'appelle désormais : département des Sciences pour l'action et le développement.
}

les phases improductives (les génisses devaient vêler à deux ans et les jeunes mâles faire la saillie dès la puberté) ; spécialiser leur aptitude pour le lait ou la viande et en faire des sortes de machines-outils de l'élevage. Bakewell et Collins, qui a appliqué les principes de ce dernier sur les bovins, créent ainsi des réseaux hiérarchiques dans lesquels l'élite entretient une clientèle qui lui offre en retour une information adaptée à ses besoins de sélection et lui permet de récupérer des meilleurs géniteurs pour son usage personnel. Ces principes de spécialisation industrielle n'ont cessé d'inspirer notre société dès la fin des guerres napoléoniennes. Les trois grands systèmes politiques que la France a connus depuis la Révolution et l'instauration de la $1^{\text {re }}$ République : la Restauration monarchique que j'associe au Second Empire, la III ${ }^{\mathrm{e}}$ République et la $\mathrm{V}^{\mathrm{e}}$ République. Ces trois systèmes ont tous essayé en vain d'industrialiser l'élevage bovin et ce n'est peut-être pas fini!

Ce sont les travaux de Bakewell que les nobles français, en rupture de cour mais en quête de réhabilitation, sont venus observer en Angleterre pour essayer de les transposer dans leurs propriétés du Nivernais et de Normandie : les sociétés d'agriculture et le concours de bœufs gras Durham de Poissy sont leurs vitrines. Ces jeunes bœufs gras intéressent bien la clientèle ouvrière de l'époque friande de viande à griller; mais, devenus adultes, ils sont incapables de tirer les charrues des paysans sur les terrains argileux. Il faudra attendre le Second Empire et Napoléon III, l'empereur des campagnes, pour qu'apparaisse une réaction paysanne organisée: des concours régionaux sont instaurés et les populations régionales de bovins y sont admises non en tant que bœufs, mais à travers des vaches et des taureaux respectivement porteurs des attributs de la fécondité. Ce mouvement régionaliste partait du Sud-Ouest, et c'est en 1859 qu'un bœuf limousin va battre les Durham.

Dans cette mouvance la bourgeoisie de la III e République va adopter ensuite l'exemple anglais en incitant à la création des livres généalogiques : le modèle animal devient une table de pointage. Bien que ces tables n'affichent pas de spécialisation productive et malgré la politique protectionniste de Jules Méline, la Belle Époque voit le développement des nourrisseurs laitiers dans la banlieue parisienne, tandis que la mécanisation bat son plein dans les fermes du Bassin parisien et pousse les éleveurs charolais, privés du débouché pour l'attelage, à finir euxmêmes leurs bœufs plus jeunes. Ainsi le mouvement de spécialisation était bien à l'œuvre sous le contrôle des sélectionneurs de la bourgeoisie des campagnes. La guerre de 14-18, puis les troubles sociaux qui vont parcourir l'Europe et le crack boursier de 1929 vont annoncer une période de rupture du mouvement d'industrialisation. Même si c'était leur dernière victoire, les paysans disposaient de plus d'atouts que les éleveurs spécialisés pour faire face à des crises du marché et de la bourse ; 
l'autarcie des années de la guerre de 39-45 marquera le pinacle de ce retour de la paysannerie, et le dorgerisme, version agraire du poujadisme dans les années 50, sera comme son chant du cygne.

La troisième vague a donc été vécue par les plus anciens d'entre nous; c'est la vague technologique dont les modèles intègrent des objets biologiques (sperme, embryon) maîtrisés par les physiologistes avec des statistiques comme les index génétiques optimaux des généticiens, des règles "diététiques » élaborées par les zootechniciens. La paysannerie est malaxée et absorbée dans des systèmes coopératifs et mutualistes destinés à mettre la « vieille Europe» au niveau du nouveau phare de l'industrialisation de l'élevage que les descendants de Bakewell ont installé aux USA. La Recherche est associée à un nouveau pouvoir qui, chez nous, prend une forme administrée avant de pouvoir s'ouvrir à la concurrence. Le reflux de cette troisième vague a débuté avec la fin des «trente glorieuses »; des expressions comme celles de néopaysans, de multifonctionalité de l'élevage marquent en effet une ambiance de fin du rêve industriel des années 1960 et de quête de retour aux réalités complexes des territoires, une quête qui vient encore du Sud.

Bref, chaque grand régime politique a poursuivi le même dessein d'industrialisation en s'appuyant sur l'état des connaissances disponibles et utiles à son époque pour devoir le corriger ensuite en faisant appel aux forces vives de sa paysannerie puis de sa ruralité. Ce que j'avais en définitive sous les yeux, entre 1960 et 1975, depuis Jouy et l'Aubrac, n'était que l'expression d'un système de vagues techniques historiques dont les prémisses remontaient à l'époque de l'industrialisation anglaise. Comme si les événements de l'histoire s'accéléraient dans la mémoire et que l'échelle de l'histoire s'y rétrécissait avec l'âge.

Il faut, bien entendu, se défier des généralisations auxquelles peut conduire une telle fresque. Les historiens de l'élevage bovin limousin montrent parfaitement comment, au milieu du XIX ${ }^{\mathrm{e}}$ siècle, la bourgeoisie de Limoges s'est opposée au courant de durhamisation pour stimuler l'élevage allaitant et le couchage en herbe de ses métairies environnantes. L'arrivée du chemin de fer et l'ouverture vers les marchés de Saint-Étienne et de Lyon vont faciliter le développement de l'élevage des veaux et des taurillons dans cette région. Cet exemple illustre bien le jeu des échelles à travers lequel se manifestent des réactions plus locales et régionales aux grandes lames de fond environnantes. Je reprendrai pour imager cela l'expression d'une géographe anglaise à propos de l'élevage des bovins au XVIII ${ }^{\mathrm{e}}$ siècle dans son pays : " le temps de l'histoire est un temps chiffonné ».

F.P. : Cette fresque historique que vous venez de dresser met en évidence des phases successives de prises de pouvoir sur la génération animale. Sans doute est-ce à partir de ces réflexions que vous avez été conduit, dans votre ouvrage, à parler de « bio-pouvoir ».
Bertrand Vissac: Dans la conduite de mes recherches, comme dans l'exercice de mes fonctions de chef de département, j'ai été confronté en permanence à ce type de tensions entre des forces poussant à l'industrialisation et d'autres tirant vers la complexification locale du développement. Ces tensions mettaient en jeu les rapports entre le savoir et le pouvoir. C'est vrai que le voisinage de Jacques Poly m'exposait à ce type de réflexion, aujourd'hui largement occulté par les multiples comités qui enserrent le scientifique dans ses rapports aux pouvoirs. Je ne pouvais en tout cas clôturer mon ouvrage sur Les Vaches de la République sans rechercher quelque facteur explicatif de ces tensions qui s'opèrent dans la génération des vaches. C'est en lisant le livre de Dreyfus et Rabinow sur Foucault que j'ai découvert, à travers l'expression de «bio-pouvoir », les similitudes qu'il y avait dans sa formulation entre l'élevage des vaches et les conduites médico-sociales. Je dois reconnaître que je n'étais pas malheureux de trouver là un support explicatif autre que celui des règles de l'économie auxquelles tout chercheur est censé devoir se conformer pour son évaluation. Avant de réifier ainsi le vivant, ne faut-il pas se référer aux écrits de Claude Bernard qui affirme, à propos de la biologie de l'organisme, que, par différence avec les corps inertes, le vivant porte en lui-même les éléments de ses propres régulations. Et si ce principe dépassait le contexte de l'organisme pour concerner aussi les populations animales et les régulations sociales auxquelles elles sont soumises?

Pour éclairer cette référence au bio-pouvoir et à ses rapports au savoir scientifique, je comparerai la situation de la génération des vaches à celle de l'amélioration du maïs. Je ne suis pas compétent pour parler de cette plante que je sais allogame; mais, comme les vaches mangent de plus en plus de maïs, je ne peux m'en désintéresser totalement!

Les chercheurs de l'Iowa observaient, vers 1900, le phénomène de consanguinité et cherchaient à connaître ses limites pour la production de lignées consanguines purifiées. Ils observent parallèlement des chutes de production mais mettent en évidence des manifestations de plus en plus spectaculaires de vigueur hybride par croisement de ces lignées. Ces observations d'un chercheur tout à fait désintéressé vont être mises à profit par un producteur local de maïs en quête de destinée politique ; il voit là la possibilité d'utiliser le travail des chercheurs pour s'approprier les lignées sans valeur intrinsèque sur le marché des semences, puisque peu productives, et pour les utiliser, ensuite, dans la production d'un hybride hautement productif. Il venait ainsi d'inventer le principe de la production de semences hybrides et le monopole auquel conduit l'accumulation et la concentration du potentiel génétique par une longue pratique préalable de la consanguinité. Même s'il s'agit de matériel vivant, je parle ici de pouvoir de type monopolistique comme dans le cas de firmes telles que Michelin, Nestlé : ce pouvoir 
s'acquiert puis se défend et se transmet, il se reproduit en cercles restreints (souvent « consanguins » eux aussi !).

Rien de tel chez les vaches : une race animale est constituée par l'association de centaines ou de milliers d'éleveurs qui, pour défendre leurs privilèges identitaires et pour utiliser les techniques collectives de sélection ou de promotion de race, doivent se regrouper et adhérer à un cahier de charges répondant à un objectif commun. Mais derrière ce qu'on peut traduire ainsi par l'expression d'un bio-pouvoir collectif dominant apparaissent des différences individuelles plus ou moins marquées de situation, d'âge, d'objectifs qui vont entraîner des résistances aux marges. Que les conditions économiques changent, que des contraintes environnementales apparaissent, cet équilibre peut évoluer plus favorablement mais aussi conduire les résistants à faire sécession en créant une sous-race, en se rattachant à un groupe voisin. La production de savoir nouveau peut aussi être rejetée ou négligée par le groupe dominant parce que ne répondant pas à son intérêt, mais elle peut intéresser un groupe de résistance pour favoriser son dessein. Notons que le savoir n'est pas que l'apanage de la science; il y a aussi des savoirs locaux tels que ceux de ces éleveurs, véritables «brocanteurs du vivant», qui ont conservé la race Maraîchine pour restaurer et entretenir les marais de l'Ouest, lorsque ont été stoppés leur assainissement et leur intensification céréalière.

Bref, le bio-pouvoir qui s'exprime dans l'élevage d'une race est fondé sur un savoir technique, un milieu géographique et une base sociale : mais cet ensemble est instable et opère à des niveaux d'organisation variés. Toute l'astuce des innovateurs est d'être d'abord les premiers, au bon endroit, et au bon moment.

Il y a une différence importante entre ces deux formes de pouvoir se rapportant pourtant toutes les deux aux mêmes bases de connaissances sur les éléments de la génération, et se conjuguant plus ou moins dans l'élevage des vaches selon les façons de produire. Dans le premier cas, les scientifiques ont produit un savoir qu'un groupe restreint a essayé de capter à son profit pour obtenir un monopole d'amélioration : ils spolient ce faisant les producteurs utilisateurs de ces semences de leur capacité de gérer et d'adapter eux-mêmes, en temps opportun, le matériel génétique à leurs milieux et objectifs ; quand ces derniers réagissent, ils ont perdu leur tradition de sélection et les moyens de la réapprendre. Nous sommes dans un schéma contraint de spécialisation industrielle. Dans le second cas, la collectivité des éleveurs se fait et se défait au gré des spécificités des exploitations, des marchés, des milieux, des techniques et de leurs évolutions. Cette voie n'ignore pas l'industrialisation, mais elle autorise le débat social et les régulations naturelles selon un processus d'alternances entre générations qui apparaît sain et normal si on veut bien accepter, ce que certains chercheurs oublient parfois, qu'il existe aussi des savoirs locaux, portés ici par des races ; si ces savoirs n'ont pas la capacité de généralisation des savoirs scientifiques dans «le monde éclairé », peut-être sont-ils plus rustiques et durables qu'eux dans «le monde d'en bas »?

F.P. : Je pense, comme vous le suggérez, que cette dynamique contrariée éclaire largement le jeu du développement technique appliqué au matériel vivant de l'agriculture. Il n'empêche que vous généralisez peut-être un peu vite vos idées d'alternance entre phases d'industrialisation et, en réaction, phases de régulations sociale et naturelle.

Bertrand Vissac : Je n'entends pas faire une démonstration à partir de 3 cycles historiques seulement, mais ce type d'alternance s'observe de façon générale et à des échelles diverses dans Les Vaches de la République. Même si j'accepte actuellement le terme mobilisateur de développement durable, je me demande s'il ne s'agit pas que d'un slogan : le développement a-t-il jamais été durable? $\mathrm{Si}$, faute de pouvoir expérimenter ce concept sur la planète Terre, on s'intéressait à quelques indicateurs du développement des sociétés, l'élevage des vaches en ferait incontestablement partie. Il me semble raisonnable de penser que nous n'avons pas fini avec cette alternance entre la voie de la spécialisation et celle du retour au local, alors même que pointe à l'horizon la vague que je ne saurais dénommer et qui sera le «cheval de bataille » de la bio-industrie. Que de rêves entretenus par la jeune génération actuelle engagée dans cette mode; mais aussi que de pain sur la planche pour la génération qui lui succédera et qui aura à faire face aux déceptions et aux adaptations nécessaires de ce formidable défi de la pensée scientifique!

F.P. : Derrière votre mobilité institutionnelle - puisque vous êtes passé d'un département à un autre -, je suis frappé de voir qu'il y a une sorte d'idée directrice tout au long de votre carrière : la génération animale. Je sais que vous vous plaisez à dire que vous avez aimé les chemins de traverse, mais en réalité, d'un bout à l'autre vous avez suivi votre idée : comprendre la génération animale, c'est-à-dire comment la société (qui est en France la République) élève ses vaches, et participer, bien sûr, à cette activité en chercheur citoyen et lucide. C'est votre fil conducteur.

Bertrand Vissac: En fait, ce n'est qu'à la fin que l'unité apparaît. Parler de génération animale au moment où les disciplines zootechniques se créaient à l'Inra eût été provocateur. Je n'avais pas été recruté pour cela. Ce n'est que lorsque ces disciplines ont été opératoires, lorsqu'elles ont disposé de relais techniques conçus par mimétisme dans les Instituts techniques, que le retour au terme global de génération animale a commencé à se poser sur le plan du savoir ; mais cette question devenait alors indissociable de celle du pouvoir auquel nous nous étions associés. 
C'est vrai que j'aurais pu soutenir, vers 1970, la thèse que Maxime Lamotte attendait de moi après la RCP Aubrac sur les rapports entre la génétique appliquée et l'écologie en élevage bovin : je me serais ainsi conformé à l'usage institutionnel et j'aurais alors écrit un mémoire de thèse sur la génération animale. L'esprit de contradiction et l'absence de formation universitaire initiale se sont conjugués pour me faire choisir la voie de « l'indiscipline » et le thème de la recherche-action. Peut-être suis-je devenu philosophe, philosophe de l'action en tout cas.

Depuis Paris, j'ai beaucoup navigué, écouté, proféré des naïvetés, mais la distance empêche aussi le chercheur appliqué de se créer des citadelles; elle l'oblige à se questionner et à rebondir dans sa pensée, ses attitudes. Cette capacité est essentielle dans le domaine de la recherche-action, qui doit aussi intégrer la réaction de l'établissement humain comme elle est apparue en Aubrac. Depuis mes deux départements successifs (la Génétique animale et le SAD), j'ai aussi beaucoup discuté, ferraillé parfois avec les puristes des disciplines. J'espère in fine qu'il restera dans la recherche des chercheurs sans thèse ou tout au moins des spécialistes de l'indiscipline. C'est peut-être ceux-là qui aideront à limiter les alternances historiques excessives du développement, à rendre le développement plus durable en évitant que la science ne décroche de la culture des gens simples. Certains taxent à tort ma posture de pessimiste, je me trouve au contraire réaliste et avant tout humaniste; c'est aux citoyens et non simplement à ceux qui sont chercheurs d'imposer leur vérité, surtout lorsque ces derniers ne mettent pas la main à la pâte des objets qu'ils influencent derrière leurs concepts.

To access this journal online: www.edpsciences.org 